\title{
S3.6
}

\section{FARMACI ANTIMALARICI E MARKERS DI RESISTENZA}

\section{Severini C., Menegon M., Majori G.}

\section{Parassitologia Umana, Istituto Superiore Sanità, Roma}

Uno dei principali ostacoli al controllo della malaria è la comparsa e la rapida diffusione della resistenza in Plasmodium falciparum ai farmaci antimalarici utilizzati per la profilassi e la terapia di questa parassitosi. Questo fenomeno costituisce una minaccia crescente per la sanità pubblica dei paesi in cui la malaria è endemica, in particolare in Africa Sub-sahariana, dove la mancanza di un farmaco efficace si traduce in un aumento della mortalità infantile. In seguito all'instaurarsi della farmaco-resistenza, i correnti protocolli terapeutici per il trattamento della malaria prevedono l'impiego di varie associazioni di farmaci dotati di diverso meccanismo d'azione. Le associazioni raccomandate prevedono combinazioni di farmaci tradizio- 
nali o di antimalarici con derivati dell'artemisinina, quali diidroartemisinina, arteetere, artemetere, artesunato, (ad esempio, atovaquone-proguanil, arteeterelumefantrina, artesunato-amodiachina, artesunatomeflochina, ecc.). Recenti indagini molecolari sull'artemisinina hanno permesso di identificare il sito di azione di questa molecola, per la quale, fino ad ora, non si registra resistenza da parte di $P$. falciparum. La conoscenza dei meccanismi cellulari alla base della farmacoresistenza riveste un ruolo centrale nelle attività di ricerca di antimalarici efficaci. Studi molecolari hanno permesso l'identificazione di mutazioni puntiformi in particolari geni del plasmodio che determinano una ridotta affinità dei farmaci per il rispettivo bersaglio. Ad esempio in P. falciparum è ben documentata l'associazione tra la presenza di mutazioni nei geni $p f d h f r$ e $p f d h p s$ e la resistenza agli antifolati, rispettivamente pirimetamina e sulfadossina. Per quanto riguarda la clorochina (CQ), correlazioni tra mutazioni di determinati geni e clorochino-resistenza (CQR) sono tuttora oggetto di studio. In P. falciparum è stato identificato recentemente un nuovo gene ( $p f c r t)$ che codifica per una proteina (PfCRT) localizzata nella membrana del vacuolo digestivo del plasmodio. In questo gene sono state identificate diverse mutazioni puntiformi ritenute cruciali per la resistenza alla CQ. La possibilità di fare definitivamente luce sui meccanismi cellulari e molecolari alla base della CQR in $P$. falciparum può rappresentare un passo importante nella conoscenza della biologia di questo parassita, più importante di quanto si possa essere portati a pensare considerando la diffusissima resistenza di falciparum alla CQ: infatti recenti studi condotti in Malawi hanno mostrato un ritorno alla sensibilità alla CQ del plasmodio in seguito alla sospensione per alcuni anni dell'impiego di questo farmaco come trattamento di prima linea della malaria. Contemporaneamente, nella stessa area, si è osservato un aumento di isolati plasmodiali $p f c r t$ wild-type rispetto a quelli portatori di mutazioni, come ulteriore prova del ruolo chiave svolto dal gene $p f c r t$ nel fenomeno della clorochino-resistenza in $P$. falciparum. 